\title{
Anabases
}

ANABASES Traditions et réceptions de l'Antiquité

$4 \mid 2006$

Varia

\section{Le monde de Bacchus}

Jean-Marie Pailler

\section{OpenEdition}

Journals

Édition électronique

URL : http://journals.openedition.org/anabases/3003

DOI : 10.4000/anabases.3003

ISSN : 2256-9421

\section{Éditeur}

E.R.A.S.M.E.

\section{Édition imprimée}

Date de publication : 1 octobre 2006

Pagination : 231-236

ISSN : 1774-4296

\section{Référence électronique}

Jean-Marie Pailler, «Le monde de Bacchus », Anabases [En ligne], 4 | 2006, mis en ligne le 01 janvier

2012, consulté le 20 octobre 2019. URL : http://journals.openedition.org/anabases/3003 ; DOI

10.4000/anabases.3003 


\section{Le monde de Bacchus}

JeAn-Marie Pailler

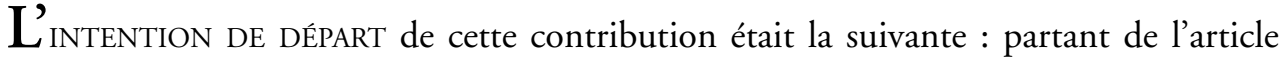
Bacchus, vérifier le degré de cohérence et de stabilité du propos tenu sur ce sujet dans l'ensemble des entrées du DAGR consacrées aux innombrables personnages et réalités traditionnellement rattachés au Dionysos grec, au Bacchus romain. Une recension approximative des corrélats mentionnés dans cet article et dans quelques autres indique une bonne centaine d'occurrences, dont la moitié environ peuvent être considérées comme pertinentes ${ }^{1}$. L'enquête inclut des titres et épiclèses du dieu (Iacchus, Zagreus, Sabazius, Liber...), des êtres divins ou semi-divins associés (Ariadne, mais non Sémélè...), des noms de membres de son «thiase » et du thiase lui-même (satyrus, Silenus, maenades...) - ainsi que d'autres groupes (orgeones, eranos...) -, avec une distinction au premier abord étrange entre thiasos et thiasus, des noms de fêtes (Dionysia, Eleusinia, Bacchanalia...), d'objets caractéristiques (cista, rhytum, cantharus, thyrsus, tympanum, oscillum), etc.

Première observation globale suggérée par cette vue panoramique : si dépassés que soient bon nombre de points de vue exprimés dans ces articles, le jeu des corrélats et des renvois mutuels qui s'y déploie permet de circuler commodément dans cet univers riche et complexe. L'actualisation à préparer devra conserver, tout particulièrement dans ce cas, cette présentation en réseau.

On peut trouver là, à première vue, une justification du programme sur lequel s'ouvrait le premier volume :

1 L'autre moitié se compose d'éléments divers, notamment les noms d'un grand nombre de dieux n'entretenant avec celui qui nous intéresse qu'une relation très lâche. 
Les noms qui servent de titres aux articles sont ceux [...] qui désignent toutes les choses de la vie publique et privée des anciens. On n'y trouvera pas de noms d'hommes ou de lieux, parce que nous ne voulions pas y mettre ce qui se trouve déjà dans les dictionnaires d'histoire et de géographie ; mais nous ne nous sommes pas davantage proposé de faire un dictionnaire de mythologie ; on n'y cherchera donc que les noms des dieux et des héros dont les types et les légendes ne devaient pas rester sans explication, à côté d'articles qui parlent de leur culte, de leurs fêtes et d'objets de toutes sortes où ils se trouvent représentés (p. 10).

L'ensemble de ces articles " bachiques " est-il cohérent ? Non, et nous verrons en quoi et pourquoi. Répondent-ils à une intention programmatique initiale suffisamment claire dans l'esprit de son ou de ses auteurs? Apparemment oui, au moins jusqu'à un certain point, et non sans une forme d'ambition " modernisante " pour l'époque. L'auteur de l'entrée Bacchus, F. Lenormant ${ }^{2}$, vient d'évoquer la pluralité " des Bacchus » attestés par Diodore de Sicile ou Cicéron, lorsqu'il écrit, dans un souci évident de clarification d'une matière menacée d'éparpillement :

Ces combinaisons présentées sous une forme entachée d'évhémérisme montrent quel besoin les anciens éprouvaient eux-mêmes d'établir des distinctions entre les dieux d'origines fort diverses qui versèrent successivement leurs fables dans la masse confuse des légendes constituant le cycle dionysiaque. Nous devons faire de même mais en procédant avec la méthode plus rigoureuse de la science moderne. Des articles spéciaux sont consacrés à Sabazius, à Zagreus, à Iacchus, ce qui permettra de nous concentrer ici sur le Dionysos proprement dit, sur le dieu de Thèbes et de Naxos (p. 600).

À vrai dire, ces deux dernières phrases incitent à la prudence, et pourraient fort bien être le produit d'une interpolation postérieure. Il se trouve en effet que les trois articles en question prennent en partie, surtout les deux derniers, le contre-pied de certaines des affirmations du Bacchus. Dans la version que nous venons de citer, le signal ainsi fait en direction de ces contributions peut exprimer la volonté de nuancer, pour le moins, l'effet produit par les propositions primitives, en ayant recours à une bibliographie postérieure, notamment de langue allemande (" la méthode plus rigoureuse de la science moderne " ?). Ainsi la brève entrée Iacchus, signée de Saglio en personne, a-t-elle été explicitement ajoutée aux fins d'une "révision " qui se réfere aux importants travaux publiés dans les années 1880-90 par Kern, Foucart, ainsi que par Höfer dans une entreprise parallèle à celle du $D A G R$, le Roscher Lexicon ${ }^{3}$. La critique

2 Bien qu'il soit décédé dès 1883 , son nom est de ceux qui apparaissent au bas d'articles publiés quelquefois longtemps après la mort de leur auteur. On mesure à quel point la concurrence de la science germanique a conduit les auteurs français du $D A G R$, dans la plupart des cas, et de manière remarquablement raisonnable pour l'époque, à intégrer les acquis de cette science admirée bien plutôt qu’à la critiquer systématiquement. 
adressée à Lenormant ressort de manière explicite du plan suivi : 1) résumé de la thèse de celui-ci sur l'Iacchus d'Éleusis ; 2) synthèse des recherches récentes qui mettent cette thèse en doute ; conclusion : "Iacchos " n'est pas, comme l'avait cru Lenormant, une appellation générale et très ancienne, mais une épiclèse purement attique et apparue tardivement. Quant à Ch. Dubois, à l'occasion des trois pages qu'il consacre à Zagreus, il conclut plus vertement encore : "Lenormant semble faire un même dieu de Sabazios et de Zagreus. Présentée ainsi, la thèse ne paraît pas admissible ».

Toute recherche historiographique sur ce terrain, particulièrement dans le cas d'un sujet aussi débordant de bibliographie dès le milieu du XIXe siècle, est donc fortement handicapée d'emblée. D'un côté, les articles du DAGR ne sont pas datés, et seule la consultation exhaustive des titres cités en note ${ }^{4}$ fournit, au mieux, un terminus post quem. De l'autre, les reprises, remords, retards de publication et éventuelles interpolations rendent, pour ce qui est du monde de Bacchus, toute proposition aléatoire.

Les remarques citées concernant le texte de Lenormant indiquent bien qu'il était largement dépassé dès la fin du XIXe siècle. Ce polygraphe (1837-1883), fils de l'historien de l'art grec Charles Lenormant (1802-1859) ${ }^{5}$ - un proche de Mérimée avec qui il fit « le voyage en Grèce »-, est plutôt spécialiste des « civilisations orientales ", comme on disait. Il est par ailleurs visiblement influencé par le courant de la mythologie naturaliste dont le leader intellectuel est alors Max Müller. C'est ainsi que, bien loin de Nietzsche ou de Rohde, Lenormant superpose volontiers le vin dionysiaque au soma de l'Inde védique, qui faisait alors l'objet d'études pionnières. Quant aux relations de Dionysos avec sa mère, la mortelle Sémélè aimée de Zeus et foudroyée par lui, voici en quels termes hyper max-müllériens (par certains côtés pré-frazériens) il en rend compte :

Le nom de Sémélè révèle en elle une personnification naturelle importante, celle du sol terrestre qui au printemps produit la végétation 6 [...] Sémélè, la terre, est fécondée par le dieu du ciel, producteur des pluies du printemps ; la manifestation de ce dieu est accompagnée de tonnerre ; le fruit que la terre produit sous l'action de son eau céleste naît imparfait, il faut qu'il grossisse et parvienne à maturité ; jusqu'à ce moment le dieu l'enveloppe dans ses brouillards qui le nourrissent et le développent... (p. 601).

Interprétation ingénieusement métaphorique de la « cuisse de Jupiter »...

4 Encore ceux-ci, dans leur grande majorité, ne comportent-ils aucune indication chronologique explicite. Dans tous ces cas, la date de parution est à restituer, parfois au prix d'un effort laborieux.

5 Le père et le fils font l'objet d'une étude synthétique de $S$. Jaubert, à paraître.

6 Dionysos est donc « fils de Gè », comme Zagreus est né de Déméter ; en Égypte, il est fils d'Isis, alors que le Sabazios phrygien serait « fils de Cybèle ». La théorie amenait à réécrire dans bien des cas l'histoire des familles divines. 
Lorsque la lecture des documents anciens vient à parâtre moins évidente à l'auteur, quelques accommodements restent possibles, et la " rigueur » des correspondances peut se voir assouplie :

"Quand les attributions du dieu se sont ensuite agrandies, quand il a représenté dans sa généralité le principe humide produisant la fertilité, le symbolisme de cette histoire ne s'est plus trouvé aussi rigoureusement précis, car alors on envisageait surtout la naissance de Dionysos comme représentant l'eau céleste qui tombe sur la terre au milieu du fracas des orages..." (ibid.). De telles remarques ne retiennent pas Lenormant, quelques lignes plus loin, de prendre ses distances à l'égard des " combinaisons factices des mythographes de profession " comme Apollodore (p. 604).

Parmi les efforts d'amélioration clairement perceptibles dans les années postérieures, outre celles qui ont déjà été signalées, il faut insister sur la succession, entre 1910 et 1914, des articles thiasos (Ch. Lécrivain, spécialiste de droit et d'histoire sociale) et thiasus (G. Nicole, historien de l'art). La distinction entre la version grecque et la version latine d'un même mot grec est sans doute unique dans les intitulés d'articles du DAGR. Elle recouvre celle qui différencie les associations humaines, dionysiaques ou non, d'une part, et d'autre part les confréries mythiques des compagnons de Dionysos. On comprend que la première entrée utilise un matériau essentiellement épigraphique, la seconde des données iconographiques, principalement les peintures des vases grecs. Scrupuleusement conduits, les deux articles s'appuient sur des études alors récentes, parfois parues dans le Roscher ou la Realencyclopädie, mais aussi sur le Cults of Greek States de Farnell et surtout les travaux en langue française de P. Foucart (marqué par l'approche sociologique), J. Girard (étude des matériaux littéraires), J. Toutain [Liber] (inspiré par le retour aux documents et leur classification), F. Cumont [SABAZIUS] (l'inventeur des religions orientales), P. Monceaux [ORPHEUS, ORPHICI ${ }^{7}$ ] (qui confronte documents chrétiens, orphiques et païens plus traditionnels)... Il est difficile de déceler à coup sûr l'origine de cet élargissement des perspectives et de ce mûrissement des méthodes. L'un et l'autre étaient " dans l'air du temps ». Mais on peut soupçonner, nous l'avons dit, le maître d'œuvre E. Saglio d'y avoir fortement contribué.

Achevons ce bref panoramique par une rapide relecture de quelques articles "bachiques ", en nous demandant quel sort pourrait leur être réservé dans le Nouveau $D @_{G R \text {. }}$

- Bacchanalia (F. Lenormant, trois colonnes) : un résumé convenable du passage de Tite-Live (livre XXXIX) concernant le scandale de 186 av. J.-C. - passage longuement cité - s’y mêle de considérations privilégiées sur des " Bacchanales étrusques " dignes,

7 Par cette dichotomie, Monceaux prend acte, notamment à partir des travaux de Kern, de la distinction progressivement établie par la science du temps entre le personnage légendaire d'Orphée et les groupes, souvent à références dionysiaques, qui se réclamaient de lui. Ces articles sont à reprendre, mais nullement à négliger. 
nous est-il dit, du " cabinet secret "! On peut estimer à juste titre que cette lecture de l'historien romain, qui accepte sans critique sa version reflétant le " tout répressif ", manque totalement d'esprit critique. Mais on en dirait autant du passage correspondant de l'Histoire Romaine de Mommsen, publiée quelques années auparavant... En revanche, Lenormant ne traite du sénatus-consulte épigraphique que par prétérition, ignorant de recherches alors en plein développement. On retiendra une notation intéressante sur le succès de ces formes religieuses en Italie du Sud, non sans s'étonner de voir la répression des Bacchanales romaines rendue responsable de « la cessation ou du moins la décadence presque complète de l'usage des vases peints " (p. 591).

En bref et en clair, le $N D @ G R$ pourra se contenter de signaler le manque d'intérêt de l'article, qui devra être entièrement refait.

- Oscillum : une première surprise vient, pour le lecteur d'aujourd'hui, du fait que la matière est ici répartie entre deux entrées : oscillum (J.-A. Hild, trois colonnes), qui examine l'usage des motifs suspendus dans le monde grec et romain (fête des aiôra à Athènes, références littéraires latines à un culte des objets attachés à des arbres...) ${ }^{8}$ et clipeus (Maurice-Albert, vingt-trois colonnes, dont la dernière intéresse notre sujet : les "boucliers suspendus»). Dans la Revue Archéologique, M. Albert venait alors de publier la première véritable étude d'ensemble sur les reliefs à double face du Musée de Naples. Regroupées, les indications fournies par les deux auteurs ne manquent pas d'intérêt.

Bilan : les données des deux articles seront à rassembler et à compléter sur deux points: l'aspect "bachique ", essentiel mais complexe (on parlerait plutôt d'univers décoratif d'inspiration bachique); une prise en compte numérique et thématique des recueils progressivement constitués. M. Albert faisait état de plusieurs dizaines d'oscilla ; cent ans plus tard les recherches de E.J. Dwyer et de J.-M. Pailler en dénombraient près de 3509 . Une étude italienne en voie de publication en compterait plus de 500. L'article à réécrire sera nettement plus fourni que l'original.

- Sabazius : ce cas est différent. Sans doute parce que l'auteur en est Franz Cumont, l'article se distingue par sa netteté d'écriture et la clarté de son recours au document. Le propos, ramassé en trois colonnes, va à l'essentiel, et en même temps on peut, on doit considérer qu'il est totalement dépassé. Plus encore que dans l'annexe "bachique » de ses futures Religions orientales, Cumont y donne libre cours à une vision " diffusionniste ", qui prétend suivre Sabazius de la Thrace à la Phrygie, puis à la Grèce et à Pergame, sans qu'il perde le caractère " essentiellement barbare " propre à " la religion

8 Le renvoi systématique au Baumcultus de Boetticher, paru en 1830, ne témoigne pas d'une bibliographie à jour.

9 Cf. J.-M. PAILLER, Les oscilla retrouvés, MEFRA 94, (1982), p. 743-820, avec la bibliographie alors disponible. 
grossière des vieilles tribus thraco-phrygiennes 10 ». On sait aujourd'hui que «l'assimilation de Sabazius avec le Yahvé Sabaoth des Israélites » est largement une vue de l'esprit. Enfin, le commentaire de l'illustration bien choisie (plaque de bronze estampée de Rome, fig. 5953) est insuffisant, de même que l'allusion trop brève à la belle étude de Blinkenberg (1904), qui concernait pourtant le témoignage capital des « mains sabaziaques".

Proposition "pédagogique " : signaler la réussite de cet article et rendre hommage à sa composition et à sa rédaction, tout en marquant ses carences évidentes. La réécriture fera une place essentielle au recueil des mains ${ }^{11}$, à la signification des symbolismes iconographiques, à la relecture des textes, à la place décidément originale de Sabazius, en relation avec Bacchus d'un côté, Cybèle de l'autre, parmi les "religions orientales".

Quelques conclusions provisoires en forme de programme paraissent simposer:

- le " réseau bachique " doit non seulement être préservé, mais consolidé, avec les diverses directions dans lesquelles il peut conduire. Ce sont une série de plaques indicatrices qui devront être dressées à partir de l'article-pivot ;

- la modernisation de l'iconographie jouera un rôle décisif dans l'actualisation de bon nombre d'entrées. Il faut pouvoir pénétrer en terre dionysiaque, au choix ou tour à tour, par le texte ou par l'image ;

- l'article-source ne peut être que celui consacré à "Bacchus ». Il conviendra tout à la fois de le raccourcir notablement, d'y intégrer ce qui est impliqué dans le DAGR sous le nom de Liber, de le rééquilibrer par conséquent du côté romain en en confiant la rédaction à deux spécialistes au moins. Enfin, il devra s'ouvrir sur deux séries de considérations historiographiques : l'une, concernant les auteurs et les tendances des années 1870-1910, a été esquissée supra. L'autre, forcément brève, devra rappeler ce que nous devons depuis un siècle à l'accroissement vertigineux de la documentation comme à l'enrichissement incessant du regard que nous portons sur Dionysos-Bacchus.

\begin{tabular}{l} 
JEAN-MARIE PAILLER \\
\hline Université de Toulouse II - Le Mirail \\
UFR d'Histoire, Arts et Archéologie \\
5, allees Antonio Machado \\
F-31058 Toulouse \\
pailler.jean-marie@wanadoo.fr
\end{tabular}

10 Nous apprenons cependant, sub fine, que « les bacchanales du dieu de la bière s'étaient peu à peu spiritualisées [sous l'Empire], conformément aux tendances religieuses de l'époque ». Cf. la publication de E.J. Lane dans les EPRO, CCIS I, The Hands, Leyde, Brill, 1983. 